\title{
Shape Effect of Piezoelectric Energy Harvester on Vibration Power Generation
}

\author{
Amat A. Basari' ${ }^{1}$, Sosuke Awaji1', Song Wang1, Seiji Hashimoto'1, Shunji Kumagai2, \\ Kenji Suto², Hiroaki Okada², Hideki Okuno², Bunji Homma², Wei Jiang3', Shuren Wang3 \\ ${ }^{1}$ Division of Electronics and Informatics, Gunma University, Kiryu, Gunma, Japan \\ ${ }^{2}$ Research and Development Department, Mitsuba Corporation, Kiryu, Gunma, Japan \\ ${ }^{3}$ School of Hydraulic, Energy and Power Engineering, Yangzhou University, Yangzhou, China \\ Email: hashimotos@gunma-u.ac.jp, s-kumaga@mitsuba.co.jp, jiangwei@yzu.edu.cn
}

Received May 2014

\begin{abstract}
Vibration energy harvesting is widely recognized as the useful technology for saving energy. The piezoelectric energy harvesting device is one of energy harvester and is used to operate certain types of MEMS devices. Various factors influence the energy regeneration efficiency of the lead zirconate titanate piezoelectric (PZT) devices in converting the mechanical vibration energy to the electrical energy. This paper presents the analytical and experimental evaluation of energy regeneration efficiency of PZT devices through impedance matching method and drop-weight experiments to different shape of PZT devices. The results show that the impedance matching method has increased the energy regeneration efficiency while triangular shape of PZT device produce a stable efficiency in the energy regeneration. Besides that, it becomes clear that the power, energy and subsequently efficiency of the triangular plate are higher than those of the rectangular plate under the condition of the matching impedance and the same PZT area.
\end{abstract}

\section{Keywords}

Vibration Power Generation, PZT Device, Impedance Matching, Energy Regeneration Efficiency

\section{Introduction}

In recent years, solutions for the environmental and energy problems correspond to the increment of power demand all over the world have become a great interest of research. One of the solution methods that highly recommended by literature is by doubling our efforts in research and development on renewable energy technology. Wind, solar, thermal, vibration and many other types of energies are always available and they do not adversely affect the environment. To date, for example, solar, thermal and wind energy have successfully been converted to usable electrical energy and used in various industrial and home appliance products [1]-[3], while vibration energy has yet to become an alternative source of energy for self-powered system. Conversion of vibration to electrical energy is possible using piezoelectric, electromagnetic and electrostatic based devices [4]. It is stated in [5] that, generally, electrostatic device is able to produce electrical energy up to $2 \%$ of efficiency while electromagnetic device according to [6], it can generate electrical power up to $1.4 \mathrm{~mW}$ with $25 \%$ efficiency. A lot of 
efforts are being introduced to optimize the energy output of every device mentioned in above. In case of piezoelectric device, analytical analysis on the vibration energy regeneration efficiency of the devices is proposed by Adachi et al. in [7]. Kong et al. in [8] presents a resistive impedance matching circuit for vibration energy harvesting. It is proven that impedance matching circuit can be utilized for optimization of energy conversion. In [9] the authors work on sensor shape design and in [10] structure of the device has been focused for the optimum energy output. Another factor that can contribute to the variation in the output level of the vibration energy harvesting system is the power conditioning circuits. This topic has been discussed in [11] and a detail summary can be found in [12].

With the recent surge of micro scale devices, piezoelectric power generation can provide a convenient alternative to traditional power sources used to operate certain types of sensors/actuators and MEMS devices. However, the energy produced by these materials is in many cases far too small to power an electrical device.

This paper presents the evaluation of vibration energy regeneration efficiency of PZT devices which is produced through impedance matching approach and by different shape of PZT devices. In addition to these, energy regeneration efficiency of PZT devices under the same PZT area and matching impedance is also evaluated analytically and experimentally.

\section{Impedance Matching of PZT Device}

\subsection{Power and Energy Generation with PZT Devices}

Impedance matching is one of the methods to increase power and energy output of PZT device power generator. Theoretically, by matching the load impedance with input impedance, maximum power can be delivered from source to the load. Prior to the evaluation on the impedance matching of the four selected bimorph PZT devices, discussion on power and energy generation by all PZT devices will be presented.

To evaluate the power and energy generation by all PZT devices, experimental setup as shown in Figure 1 was built. The experiments were conducted based on the experimental conditions as listed in Table 1. Relation
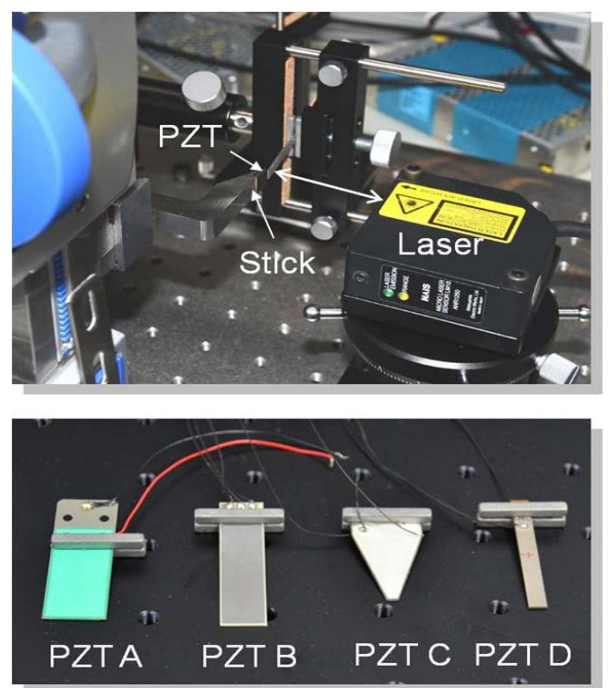

Figure 1. Experimental setup and PZT devices.

Table 1. Experimental conditions.

\begin{tabular}{cc}
\hline Parameters & Details \\
\hline 1) Input signal & Step signal \\
2) Amplitude & $0.5 \mathrm{~mm}$ \\
3) Measurement signals & PZT voltage and displacement of PZT device’s free end \\
4) Sampling time & $0.2 \mathrm{~ms}$ \\
5) Displ. resolution & $3.5 \mu \mathrm{m}$ \\
6) ADC resolution & $16 \mathrm{bit}$ \\
\hline
\end{tabular}


ship between the resonant frequency, matching impedance and energy is described in the following section.

\subsection{Impedance Matching for Maximum Power and Energy Generation}

In the previous section, discussion on power generation by the PZT devices with fixed load resistor of $10 \mathrm{k} \Omega$ has been presented. This section will discuss the impedance matching of PZT devices so that maximum power and energy can be generated. For PZT device A, load resistor was increased gradually from $100 \Omega$ to $3 \mathrm{M} \Omega$. The results of power dissipation by each resistor are shown in Figure 2.

From the figure, maximum power was dissipated by the $12.8 \mathrm{k} \Omega$ resistor and maximum energy was generated when resistor is $100 \mathrm{k} \Omega$. Notice that the maximum power and energy are dependent on different value of resistors. From this observation we know that PZT device A has two resonant frequencies.

Next, power spectral density (PSD) of $100 \mathrm{k} \Omega$ resistor was plotted. It is clearly can be seen in Figure 3, the resonant frequency of PZT device A appears at two different frequencies; at $39 \mathrm{~Hz}$ and $586 \mathrm{~Hz}$. For PZT device $\mathrm{B}, \mathrm{C}$ and D, same experiments were conducted and their dissipated voltage's power spectral densities were analyzed. Maximum power and energy for each PZT device were found to be dependent on the load resistors.

\subsection{Energy Regeneration Efficiency}

To evaluate the energy regeneration efficiency of each PZT device, experimental set up as shown in Figure 4 was constructed and ratio of output to input energy was calculated. Note that one end of the cantilever beam was clamped and the other end was free and attached with mass $m=0.1 \mathrm{~kg}$ by string. At the rest position, the displacement of the free end is $x_{0}$. The displacement of the free end after the string is cut was measured using laser measuring device while the voltage drop was measured using voltmeter.

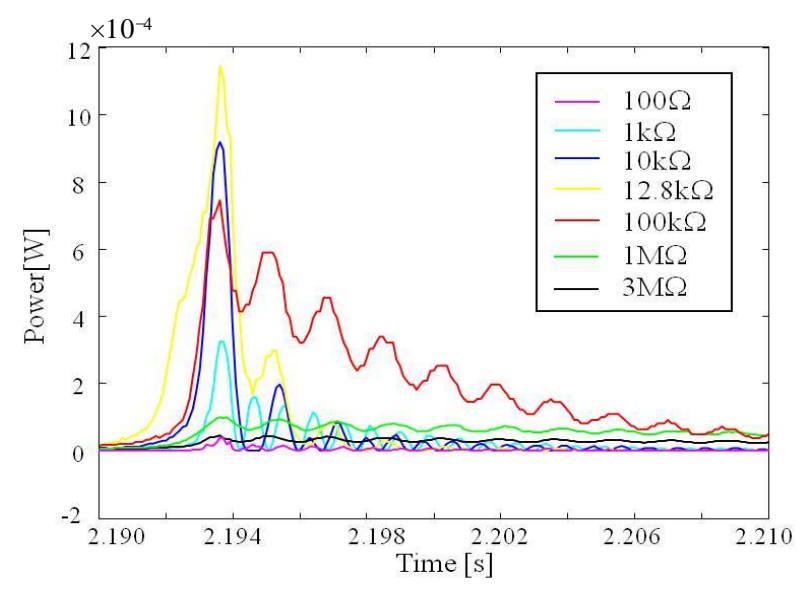

Figure 2. Power dissipation by resistors-PZT device A.

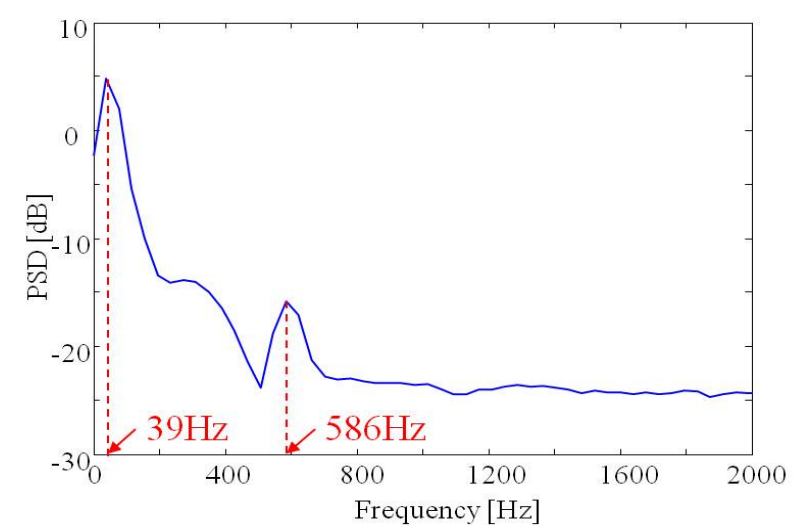

Figure 3. Voltage power spectral density of $100 \mathrm{k} \Omega$ resistor. 
Without considering the impedance matching results, the load resistor is set to $10 \mathrm{k} \Omega$ for all PZT devices. Input energy, output energy and energy efficiency were calculated based on the Equations (1)-(3). Results are summarized in Table 2.

$$
\begin{gathered}
W_{i}=\int F d x \approx \frac{1}{2} m g x_{0} \\
W_{o}=\frac{1}{R} \int V^{2} \mathrm{~d} t \\
\eta=\frac{W_{o}}{W_{i}} \times 100 \%
\end{gathered}
$$

Now, based on the impedance matching results, load resistor was replaced with the impedance matching resistor that produced maximum energy for each PZT device. The displacement and voltage drop were measured and recorded. As shown in Table 2, it is clear that energy regeneration efficiency has increased between $4 \%$ to 88\% for all PZT devices with the impedance matching resistor.

\section{Energy Regeneration Efficiency of Different Shape PZT Devices}

This section will discuss the effect of the shape of PZT device to the energy regeneration efficiency. In the previous section, four different size PZT devices have been tested. Basically, PZT device A, B and D have the same shape. Thus, in this section, to evaluate the effect of the PZT shape on the energy regeneration efficiency, PZT device $\mathrm{A}$ and $\mathrm{C}$ which having different shapes were used.

\subsection{Stress, Deflection and Elastic Energy Analysis}

Dimension of PZT device C in the cantilever beam structure is shown in Figure 5. In this figure, $W$ represents the concentrated load that applied on the free end of the cantilever beam. Theoretically, in bending mode, maximum stress is calculated using Equation (4).

$$
\sigma_{\max }=\frac{M}{Z}=-\frac{6 W_{x}}{b h^{2}}
$$

where, $M$ is bending moment, $Z$ is section modulus, $x$ is distance from the load, $b$ is the width and $h$ is the thickness of the PZT plate. From Equation (4), thickness $h$ is a fixed value, and if ratio of $x / b$ is also fixed, the maximum stress at all points of the PZT plate will become constant. Based on this fact, different from PZT device A which having the maximum stress at the clamped point, PZT device $C$ is the one with the constant value of maximum stress at all points of the plate for which the width $b$ will increase as distance from the free end $x$ in

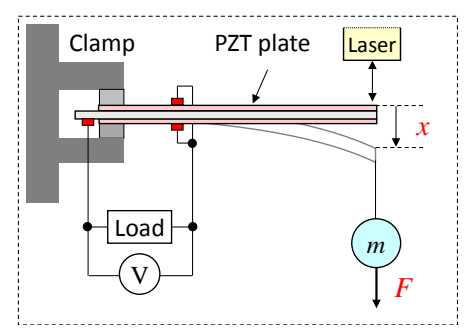

Figure 4. Experimental setup for energy measurement.

Table 2. Energy regeneration efficiency.

\begin{tabular}{cccc}
\hline PZT & Efficiency with 10k $\Omega$ resistor [\%] & Efficiency with impedance matching resistor [\%] & Increment in efficiency \\
\hline A & 3.0 & 5.4 & $80 \%$ \\
B & 4.8 & 5.0 & $4 \%$ \\
C & 3.1 & 4.9 & $58 \%$ \\
D & 1.7 & 3.2 & $88 \%$ \\
\hline
\end{tabular}




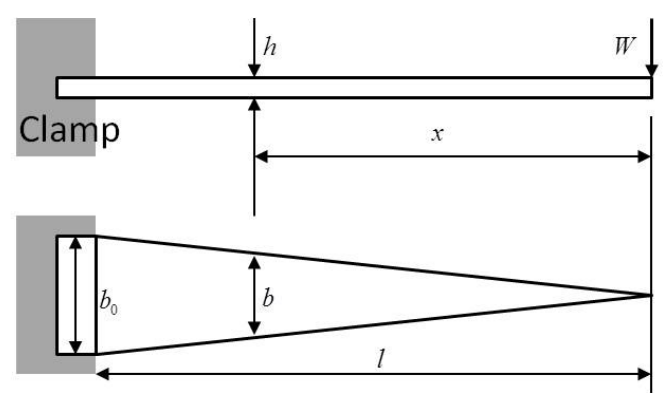

Figure 5. Cantilever beam of PZT device C.

creases.

Further analysis on deflection and elastic energy experienced by both PZT devices was performed. Based on the above analysis, the curvature of PZT device $\mathrm{C}$ will be a constant value when concentrated load is applied. Moreover, if compared to PZT device A, the deflection angle of the PZT C is double and this will increase its deflection and the input energy $W_{i}$, to 1.5 times of that the PZT device A has. The equation that denoted the input energy is shown in equation 1. By looking at the equation, it can be expected that energy regeneration efficiency will become better than what PZT device A has. Another merit point that PZT device C has here is if we look to its volume. By having the same durability, the cost also can be reduced as its volume is half of the PZT device A.

Meanwhile, in terms of elastic energy per unit volume for PZT device A and C, they are denoted by Equations (5) and (6) respectively.

$$
\begin{aligned}
& U_{A}=\frac{1}{2} W y_{0} \frac{1}{V_{A}}=\frac{\sigma^{2}}{18 E} \\
& U_{C}=\frac{1}{2} W y_{0} \frac{1}{V_{C}}=\frac{\sigma^{2}}{6 E}
\end{aligned}
$$

where $y_{0}$ is the maximum deflection, $E$ is the Young's modulus and $V$ is the volume. As mentioned in above, with 1.5 times of maximum deflection and half of the volume, it is clearly can be seen from the equation that PZT device $C$ will be able to absorb 3 times greater externally applied elastic energy than of that the PZT device A would absorb.

\subsection{Experimental Analysis of Shape Effect}

Energy regeneration by PZT device is very much related to the stress that can be generated by the PZT device where we know that deflection is the source for the stress. An increment in the deflection simply can be done by increasing the concentrated load.

Therefore, to observe the effects of the shape of PZT plate to the energy regeneration efficiency, an experimental setup as shown in Figure 4 was constructed. The experiments were conducted with load mass which was used as the varying parameter. It was increased gradually from $50 \mathrm{~g}$ to $350 \mathrm{~g}$. The output was connected to the impedance matching resistor of each case. Input and output energy data were measured and recorded.

From Figure 6, it can be seen that both input energy of PZT device A and C increase with quadratic function of the load mass. Besides that, the output energy of PZT device C, as shown in Figure 7 also increases with the same function. Contradict to the rest, the output energy generated by the PZT device A is saturated at low level even the load mass is increased. In terms of energy regeneration efficiency, as can be seen in Figure 8, the PZT device $\mathrm{C}$ marked a stable efficiency at about 5\% as the load mass increases when compared to that of the PZT device A.

\subsection{Shape Effect on Same PZT Area}

To experimentally evaluate the shape effects, as illustrated in Figure 9, PZT devices were attached on the same size of rectangular and triangular plates. The length a, b and c are 59, 30 and $10 \mathrm{~mm}$, respectively. Experimental analyses with step signal input were first carried out and matching impedances for maximum power and energy 


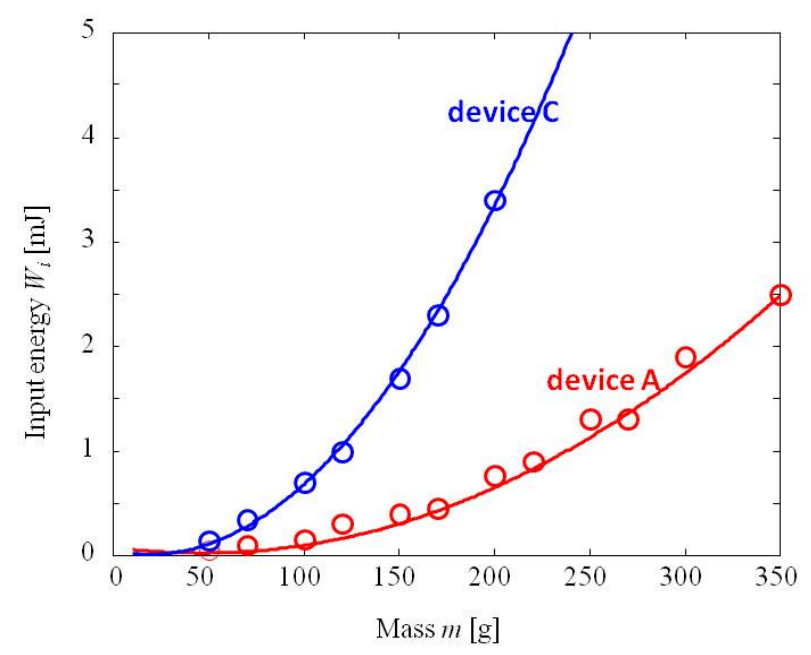

Figure 6. Input energy of PZT cantilever beam versus load mass.

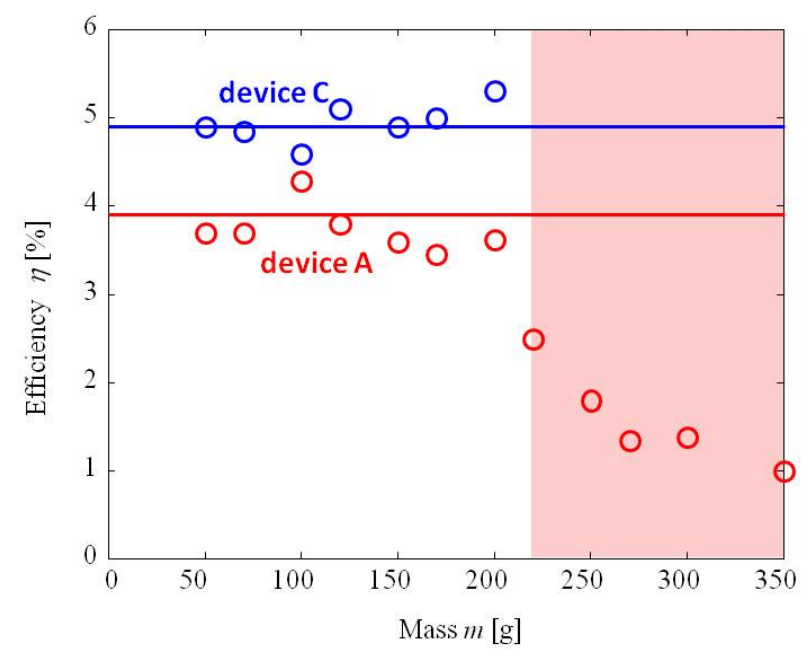

Figure 7. Output energy of PZT cantilever beam versus load mass.

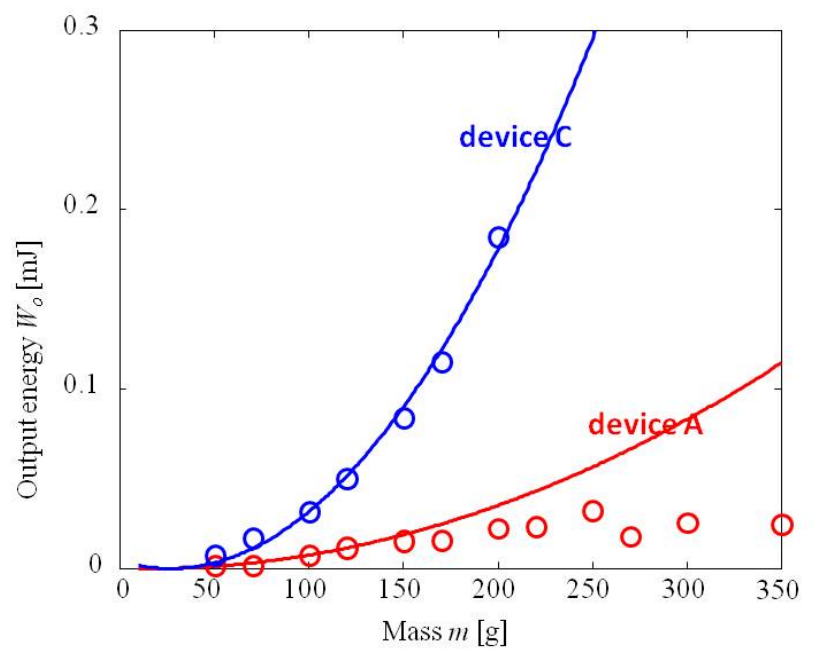

Figure 8. Energy regeneration efficiency of PZT cantilever beam versus load mass. 
generation were derived. Initially, free end of the plates were deflected by $3 \mathrm{~mm}$ from its origin and then, displacement of the free end and voltage across the load were measured.

The results are as follows; For the triangular plate, resonant frequencies of $185 \mathrm{~Hz}$ and $372 \mathrm{~Hz}$ generate maximum energy and power with $4 \mathrm{k} \Omega$ and $2 \mathrm{k} \Omega$ matching impedances respectively. On the other hand, the resonant frequency for the rectangular plate is $104 \mathrm{~Hz}$ and its matching impedance is $10 \mathrm{k} \Omega$. Since the resonant frequencies of the triangular plate are higher than that of the rectangular plate, the matching impedances for the triangular plate are smaller than that for the rectangular plate. For the triangular plate, two resonant frequencies were measured. As a result, two matching impedances are obtained.

Next, by using the obtained matching impedances, energy generation efficiency of the plates were experimentally investigated. This time, mass was attached by string to the free end of the plates and after the string was cut, its displacement and instantaneous voltage across the load were measured. The input and output energy for each mass can be calculated from (1) and (2). Figure 10 and Figure 11 show the input and output energy plots respectively. It can be seen from the plots that both curves are increase quadratically with mass.

Moreover, the calculated efficiency by (3) is shown in Figure 12. From Figure 12, since the resonant fre-

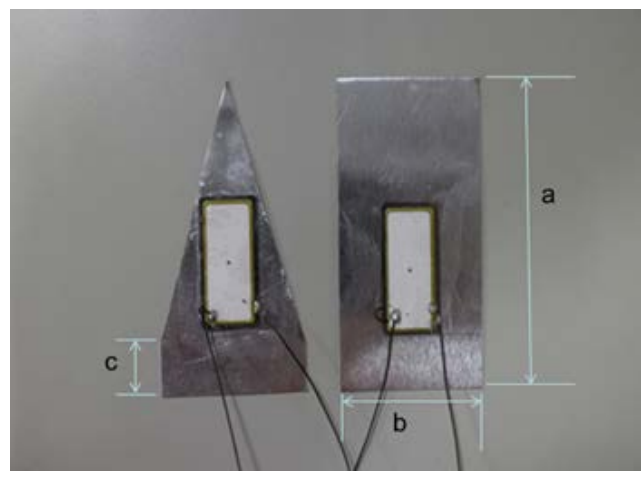

Figure 9. Picture of triangular and rectangular plates.

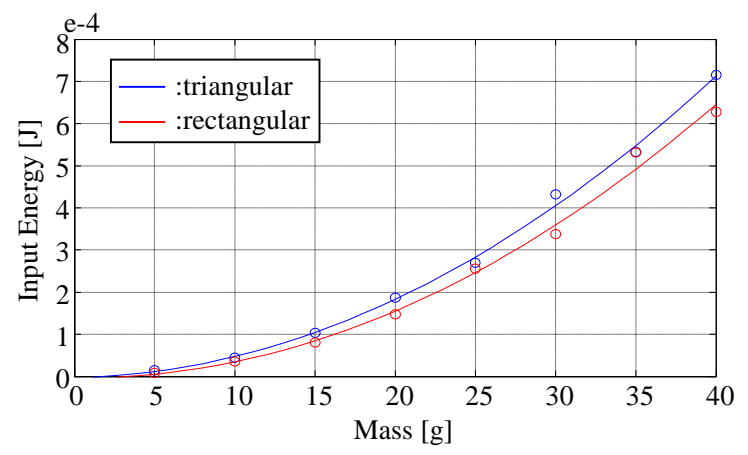

Figure 10. Input energy versus load mass.

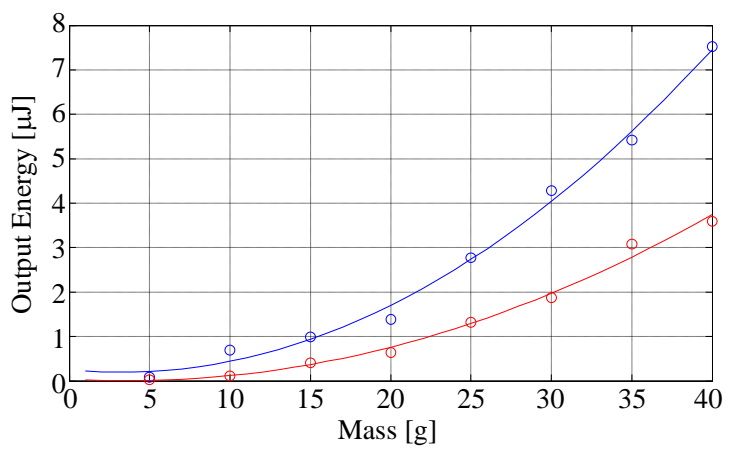

Figure 11. Output energy versus load mass. 


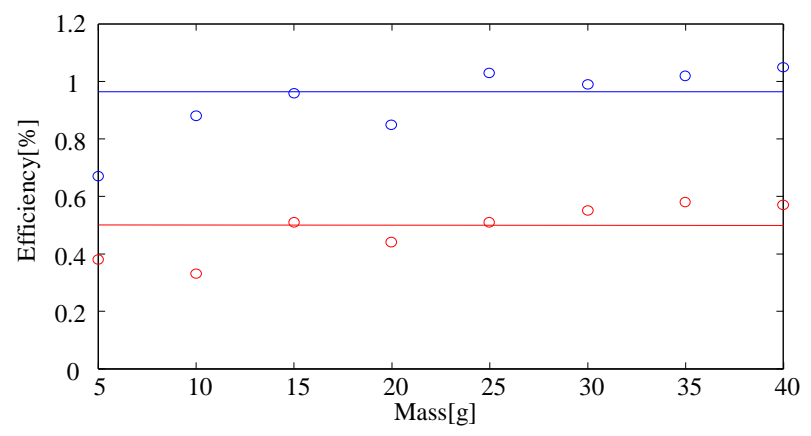

Figure 12. Efficiency comparison.

quency of the triangular plate is higher than that of the rectangular plate, the matching impedances are smaller. As a result, current, power, energy and subsequently efficiency of the triangular plate are higher than that of the rectangular plate.

\section{Conclusion}

This paper has successfully evaluated how impedance matching, shape of devices, concentrated mass and forced vibration contribute to the variation of the energy regeneration efficiency of PZT devices. Input and load impedance matching has shown an increment in the energy regeneration efficiency to between $4 \%$ and $88 \%$. On the other hand, a stable 5\% energy regeneration efficiency was produced by a triangular plate of PZT device while the rectangular plate's efficiency has worsened as load mass increased.

Further analysis on the output of triangular PZT device shows that power, energy and subsequently efficiency of the triangular plate are higher than those of the rectangular plate under the condition of the matching impedance and the same PZT area.

\section{References}

[1] Rizza, J.J. (2013) Solar-Driven LiBr/ $\mathrm{H}_{2} \mathrm{O}$ Air Conditioning System with a R-123 Heat Pump Assist. Journal of Solar Energy Engineering, 136, 1-5. http://dx.doi.org/10.1115/1.4024741

[2] Beshore, D.G., Jaeger, F.A. and Gartner, E.M. (1979) Thermal Energy Storage/Waste Heat Recovery Application in the Cement Industry. Proceedings of First Industrial Energy Technology Conference, 747-756.

[3] Hatziargyriou, N. and Zervos, A. (2004) Wind Power Development in Europe. Proc. of the IEEE, 1765-1782.

[4] Hulst, R.D., Sterken, T., Puers, R., Deconinck, G. and Driesen, J. (2010) Power Processing Circuits for Piezoelectric Vibration-Based Energy Harvesters. IEEE Transaction on Industrial Electronics, 57, 4170-4177. http://dx.doi.org/10.1109/TIE.2010.2044126

[5] Miranda, J.O.M. (2004) Electrostatic Vibration-to-Electric Energy Conversion. PHD thesis, Massachusetts Institute of Technology.

[6] Robert, G. and Radu, O. (2011) Harvesting Vibration Energy by Electromagnetic Induction. Electrical Engineering Series, 7-12.

[7] Adachi, K. and Sakamoto, T. (2012) Study on Energy Transfer Efficiency Analysis of Cantilever Type of Piezocomposit Vibration Energy Harvester. The Japan Society of Mechanical Engineers, 271-281.

[8] Friswell, M.I. and Adhikari, S. (2010) Sensor Shape Design for Piezoelectric Cantilever Beams to Harvest Vibration Energy. Journal of Applied Physics, 108, 1-7.

[9] Hashimoto, S., Nagai, N., Fujikura, Y., Takahashi, J., Kumagai, S., Kasai, M., Suto, K. and Okada, H. (2012) Multi-Mode Vibration-Based Power Generation for Automobiles. Proceedings of the 2012 IEEE-IAS Annual Meeting, 1-5.

[10] Young, M. (1989) The PWM Strategy on DC-DC Converter. IEEE Journal of Industry Applications, 28, 123-129.

[11] Eason, G., Noble, B. and Sneddon, I.N. (1995) On Certain Integrals of Lipschitz-Hankel Type Involving Products of Bessel Function. IEEE Transaction on Power Electronics, 247, 529-551.

[12] Maxwell, J.C. (2010) A Treatise on Electricity and Magnetism. IEEE Transaction on Industry Applications, 589, 6873. 